\title{
Population Structure of the Gibberella fujikuroi Species Complex Associated with Rice and Corn in Korea
}

\author{
Ji-Hye Kim¹, Mi-Ran Kang ${ }^{1}$, Hee-Kyoung Kim¹, Seung-Ho Lee ${ }^{2}$, Theresa Lee ${ }^{2}$ and Sung-Hwan Yun ${ }^{1 *}$ \\ ${ }^{I}$ Department of Medical Biotechnology, Soonchunhyang University, Asan 336-745, Korea \\ ${ }^{2}$ Microbial Safety Team, National Academy of Agricultural Science, RDA, Suwon 441-707, Korea
}

(Received on September 1, 2012; Revised on September 20, 2012; Accepted on September 23, 2012)

Several species belonging to the Gibberella fujikuroi species $(G f)$ complex are commonly associated with rice and corn, not only causing serious diseases, but also producing fumonisins, a group of mycotoxins harmful to animals and humans. To characterize the population structure of the putative fumonisin-producing $\boldsymbol{G} \boldsymbol{f}$ complex in Korea, we obtained 276 candidate isolates from rice and corn harvested in 2009 and 2010 by diagnostic polymerase chain reaction with several specific primer sets. Phylogenetic trees were constructed using multilocus sequences (combined $R P B 2$ and $E F 1 A$, totaling $1.6 \mathrm{~kb}$ ) from these isolates. Among the 135 isolates from rice, F. fujikuroi (teleomorph: $G$ fujikuroi; $59.3 \%$ ) and $F$. proliferatum ( $G$ intermedia; $13.3 \%$ ) were predominant, followed by $\boldsymbol{F}$. concentricum (5.9\%). Additionally, twentyfive $(18.5 \%)$ rice isolates belonged in a distinct subclade of $F$. commune, a non-member of the $G \boldsymbol{f}$ complex. In contrast, $F$. verticillioides was the most predominant species $(38.3 \%)$ among the 141 corn isolates, and followed by $F$. fujikuroi $(27.7 \%)$, F. proliferatum $(14.9 \%)$, F. subglutinans $(7.1 \%)$, and $F$. concentricum $(2.8 \%)$. A single mating type (MAT1-1) was found predominantly among the $\boldsymbol{G} f$ complex isolates examined. Possible distinct subclades were detected within the populations of $F$. fujikuroi and $F$. proliferatum; however, this needs further confirmation. This is the first reported population-level characterization of putative fumonisin-producing $\boldsymbol{G} \boldsymbol{f}$ complex associated with rice and corn in Korea.

Keywords : fumonisin, Gibberella fujikuroi species complex, mating-type distribution, population structure, species composition

The genus Fusarium is one of the most important fungal isolates associated with rice and corn because some members of this group, such as the F. graminearum species complex and Gibberella fujikuroi species complex, are mycotoxin producers as well as plant pathogens. The G. fujikuroi species

\footnotetext{
* Corresponding author.

Phone) +82-41-530-1288, FAX) +82-41-530-3085

E-mail)sy14@sch.ac.kr
}

complex, a monophyletic lineage, consists of at least nine biological species, formerly designated as mating populations (MPs) (Britz et al., 1999; Leslie 1999; Leslie et al., 2004), and numerous additional Fusarium anamorphs that are assigned to phylogenetically distinct species (Nirenberg and O'Donnell 1998; O'Donnell et al., 1998; O'Donnell et al., 2000).

The Fusarium species in the G. fujikuroi species complex are distributed worldwide and cause serious diseases in a wide variety of agricultural crops (Leslie 1999). These species also produce a variety of toxic secondary metabolites and mycotoxins, including gibberellic acid (Cerda-Olmedo et al., 1994), moniliformin (Marasas et al., 1986), and fumonisins (Rheeder et al., 2002). Among these, fumonisins, a group of polyketide-derived mycotoxins, have gained considerable attention because they have been associated with esophageal and liver cancer (Desjardins, 2006).

To date, at least 15 Fusarium species, most belonging to the G. fujikuroi species complex, are known to produce fumonisins using a biochemical pathway catalyzed by a polyketide synthase (FUM1) (Proctor et al., 1999). Only a few species outside the G. fujikuroi species complex, such as $F$. oxysporum, have the capacity for fumonisin production (Rheeder et al., 2002).

As both rice grains and corn kernels are capable of harboring the toxigenic G. fujikuroi species complex during the growing season, monitoring the fumonisin-producing Fusarium species on these substrates is important. Several species within the G. fujikuroi species complex are known to be frequently associated with rice and corn.

Three fumonisin-producing species, F. fujikuroi (MP-C, teleomorph: G. fujikuroi), F. proliferatum (MP-D, G. intermedia), and $F$. verticillioides (MP-A, G. moniliformis), have been isolated from rice plants showing bakanae symptoms in Asia, Africa, Europe, and the United States (Amatulli et al., 2010; Carter et al., 2008; Desjardins et al., 2000; Desjardins et al., 1997; Hsuan et al., 2011; Wulff et al., 2010; Zainudin et al., 2008). The incidence of the latter was relatively lower than the first two species. $F$. verticillioides is the most prevalent species on corn worldwide (Chulze et 
al., 2000; Kedera et al., 1999; Leslie 1991; Rahjoo et al., 2008).

Despite many studies worldwide, limited information is available on the population structure of G. fujikuroi species complex in these crops in Korea (Choi et al., 2009; Park et al., 2001). The occurrence of fumonisins in rice and corn samples has been reported in Korea (Chung and Kim, 1995; Kim et al., 1998; Seo and Lee, 1999).

Identification of these species has relied on time-consuming methods such as morphological comparisons, sexual crosses to tester strains, mycotoxin profiling, vegetative compatibility grouping, random amplification length polymorphic DNA (RAPD), and amplified fragment length polymorphism (AFLP) analyses. Even these procedures, however, do not guarantee precise species identification within the $G$. fujikuroi species complex.

As an alternative, delineation of phylogenetic species within the species complex using DNA sequence data has been widely used because it revealed perfect concordance between phylogenetic species within the phylogenetic trees and mating populations (O'Donnell et al., 1998; O'Donnell et al., 2000). The objectives of the present study were to determine the species composition and genetic diversity in putative fumonisin-producing G. fujikuroi species complex associated with rice and corn in Korea.

\section{Materials and Methods}

Fungal isolation. Husked seeds of rice were collected from 39 sampling sites in eight provinces (Gyeonggi-do, Gangwondo, Chungcheongnam-do, Chungcheongbuk-do, Jeollanamdo, Jeollabuk-do, Gyeongsangnam-do, and Gyeongsangbukdo) in Korea. Corn kernels were collected from nine sites in six provinces, but mostly in Gangwon-do where is major corn-cultivating area. Both grain samples were harvested in 2009 and 2010. Fungal contaminants were recovered from surface-sterilized grains as described previously (Son et al., 2011), from which Fusarium spp. were initially selected based on colony morphology. Further diagnostic polymerase chain reaction (PCR) using genomic DNAs from the Fusarium spp. collection identified putative isolates belonging to the Gibberella fujikuroi species complex. They were stored in $25 \%$ glycerol at $-80^{\circ} \mathrm{C}$ and maintained on potato dextrose agar (PDA; Difco Laboratories, Detroit, MI, USA). For genomic DNA extraction, fungal isolates were grown in $50 \mathrm{~mL}$ of PD broth at $25^{\circ} \mathrm{C}$ for 3 days with shaking (150 rpm). All of the G. fujikuroi species complex isolates obtained in this study (Table S1) are available on request; several isolates are also deposited in KACC (Korean Agricultural Culture Collection, Suwon, Korea, http:// www.genebank.go.kr, no. 45818-45826).
Nucleic acid manipulation and PCR. Fungal genomic DNA was extracted as described previously (Hong et al., 2010). All primers used for PCR amplification were synthesized by Bioneer Corporation (Daejeon, Korea). The PCR amplification was performed with the conditions described previously (Hong et al., 2010; Kang et al., 2011). For diagnostic PCR of the G. fujikuroi species complex, three specific primer sets were used: VER1/VER2 and PRO1/PRO2 as $F$. verticillioides- and $F$. proliferatumspecific primer sets, respectively (Mule et al., 2004), and rp32/rp33 for the FUM1 gene, responsible for fumonisin biosynthesis (Proctor et al., 2004). For amplification of $E F 1 A$ (encoding translation elongation factor 1-alpha) and $R P B 2$ (RNA polymerase, second largest subunit), the primer sets fRPB2-7cF/fRPB2-11aR (Liu et al., 1999) and EF1/ EF2 (O'Donnell et al., 1998), respectively, were used. Mating type $(M A T)$ of the fungal isolates was established by PCR using primer pairs specific for two MAT genes (MAT1-1 and MAT1-2) (Steenkamp et al., 2000).

Phylogenetic analysis. Amplified products of EF1A or $R P B 2$ were either cloned into the pGEMT vector (Promega, Madison, WI, USA) or subsequently purified for nucleotide sequencing. All nucleotide sequences for each isolate are available on request and some were deposited in GenBank (accession numbers: JN968201-JN968212, JX867934JX867953 \& KC121007-KC121025). Additionally, both EF1A and RPB2 sequences of several Fusarium species deposited in the National Center for Biotechnology Information (NCBI; http://www.ncbi.nlm.nih.gov) were used as reference sequences for a phylogenetic analysis (Table S2). Sequences were edited with Lasergene (ver. 6.0; DNASTAR, Madison, WI, USA) and aligned using ClustalW (Thompson et al., 1994). Maximum parsimony (MP), neighbor-joining (NJ), and unweighted pair group method with arithmetic mean (UPGMA) analyses were performed using MEGA (ver. 4.02) with 1,000 bootstrap replications. The DNA sequences of $F$. equiseti and $F$. incarnatum were used as outgroups (Table S1).

\section{Results}

Isolation of putative fumonisin-producing Fusarium isolates. Putative isolates belonging to the G. fujikuroi species complex were selected from the collection of Fusarium isolates recovered from rice and corn grain samples. Fungal identification was performed based on PCR amplification with primer sets known to be specific to $F$. proliferatum and F. verticillioides, and FUM1, a polyketide synthase gene responsible for fumonisin production. A fungal isolate from which an expected-size DNA frag- 


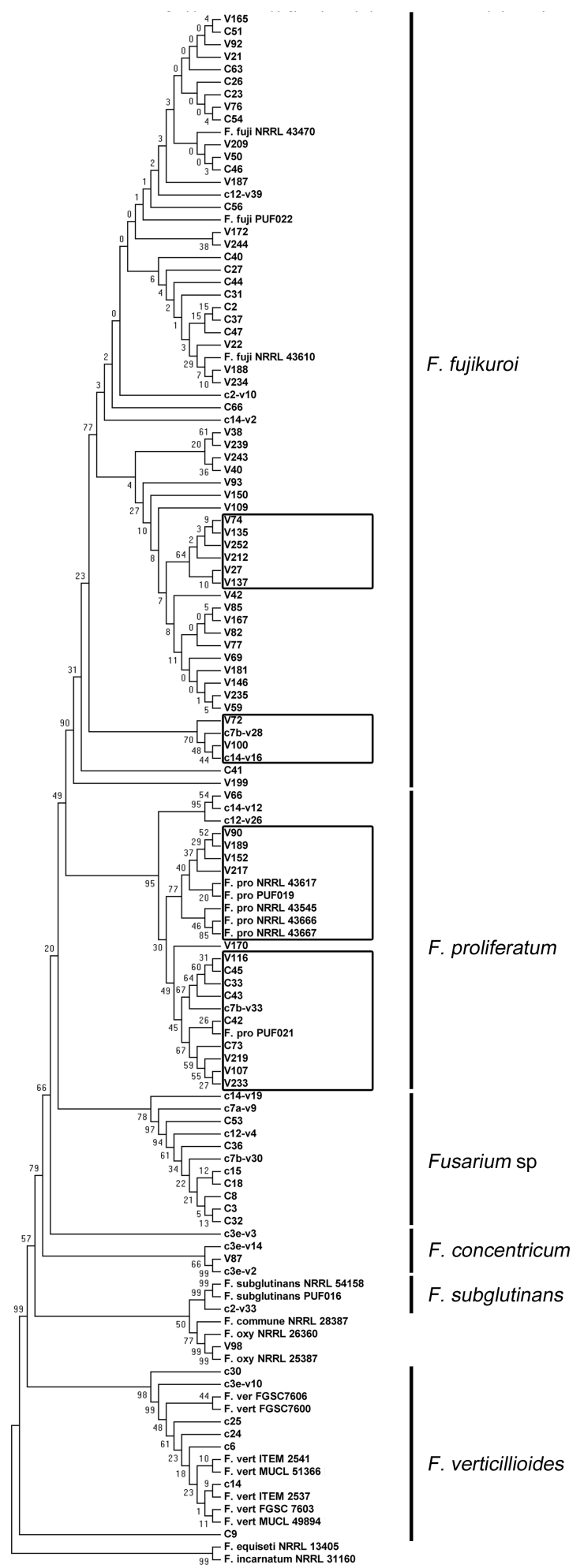

Fig. 1. Phylogenetic tree constructed with the UPGMA method using combined nucleotide sequences of EF1A and RPB2 from the Fusarium isolates obtained in 2009. Names starting with V and $\mathrm{C}$ (or c) represent the isolates recovered from rice and corn, respectively. The isolates belonging to the putative subclades within each species described in the text are indicated by rectangular boxes.

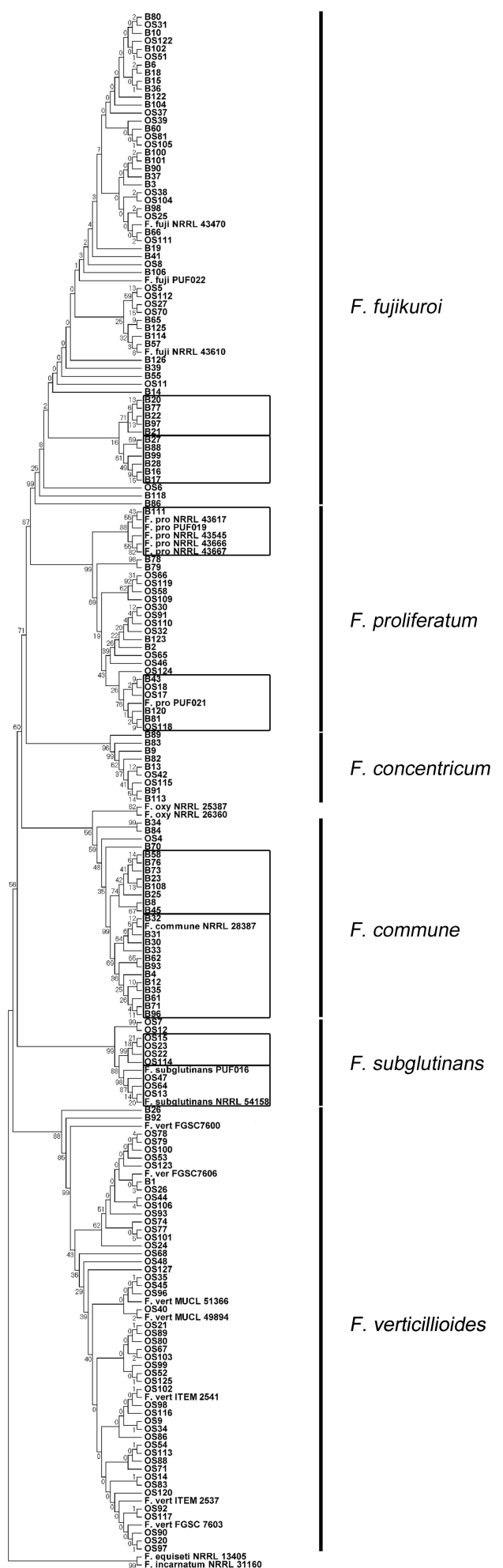

Fig. 2. Phylogenetic tree constructed with the UPGMA method using combined nucleotide sequences of $E F 1 A$ and $R P B 2$ from the Fusarium isolates obtained in 2010. Names starting with B and OS represent the isolates recovered from rice and corn, respectively. The isolates belonging to the putative subclades within each species described in the text are indicated by rectangular boxes. 
although their species identification remained unclear, seemed to be closely related to the $F$. proliferatum clade (Fig. 1). Overall, among the G. fujikuroi species complex isolates from rice, $F$. fujikuroi $(47.1 \%)$ and $F$. proliferatum $(16.8 \%)$ were predominant, followed by $F$. concentricum $(7.5 \%)$ and $F$. verticillioides $(0.9 \%)$, whereas $F$. verticillioides was predominant $(42.2 \%)$ among the corn isolates, followed by F. fujikuroi (30.5\%), F. proliferatum (16.4\%), F. subglutinans $(7.8 \%)$, and $F$. concentricum $(3.1 \%$; Tables $1, \mathrm{~S} 1)$.

Genetic diversity within species. The presence of distinct subclades within the species clades of the G. fujikuroi species complex was examined in the phylogenetic trees constructed using UPGMA (Figs. 1, 2) and NJ methods (data not shown), respectively. In the F. fujikuroi clade, two subclades one consisting of six isolates (including V74 and $\mathrm{V} 135$ ) and the other of four isolates (including V72) were found in the 2009 trees (with $64 \%$ and $70-73 \%$ BS in the UPGMA and NJ trees, respectively; Fig. 1), and two subclades in the 2010 trees, consisting of five isolates (B20, B77, B22, B97, and B21, with 71\% UPGMA and 63\% NJ $\mathrm{BS}$ ), and six isolates (B27, 88, 98, 28, 16, and 17, with $61 \%$ UPGMA and $76 \%$ NJ BS; Fig. 2), respectively. Note that most of the isolates belonging to these subclades were recovered from rice samples only. In the $F$. proliferatum clades in both 2009 and 2010, two distinct subclades were identified; one was the subclade to which the five $F$. proliferatum reference strains as well as the field isolates (all from rice) were grouped, and the other was for the one remaining reference strain, which were supported with higher than $67 \%$ BS in both the UPGMA and NJ trees. Similarly, at least two subclades were found in the $F$. subglutinans clades in the 2010 trees; one included two reference strains of F. subglutinans and three isolates (OS47, OS64, and OS13), and the other consisted of four field isolates, which were supported with high BS $(>98 \%)$ in both the
Table 1. Species composition of the putative fumonisin-producing Fusarium isolates from Korean rice and corn in 2009-2010

\begin{tabular}{|c|c|c|c|c|}
\hline \multirow[b]{3}{*}{ Species $^{\mathrm{a}}$} & \multicolumn{4}{|c|}{ Number of isolates identified for each species } \\
\hline & \multicolumn{2}{|c|}{2009} & \multicolumn{2}{|c|}{2010} \\
\hline & rice & corn & rice & corn \\
\hline \multicolumn{5}{|c|}{$\begin{array}{l}\text { members of the } \\
\text { G. fujikuroi species complex }\end{array}$} \\
\hline F. fujikuroi & 38 & 21 & 42 & 18 \\
\hline F. proliferatum & 10 & 8 & 8 & 13 \\
\hline F. verticillioides & 0 & 7 & 1 & 47 \\
\hline F. concentricum & 1 & 2 & 7 & 2 \\
\hline F. subglutinans & 0 & 1 & 0 & 9 \\
\hline subtotal & 49 & 39 & 58 & 89 \\
\hline \multicolumn{5}{|l|}{ other species } \\
\hline F. commune & 0 & 0 & 25 & 1 \\
\hline F. oxysporum & 1 & 0 & 0 & 0 \\
\hline unidentified & 0 & 12 & 2 & 0 \\
\hline subtotal & 1 & 12 & 27 & 0 \\
\hline total & 50 & 51 & 85 & 90 \\
\hline
\end{tabular}

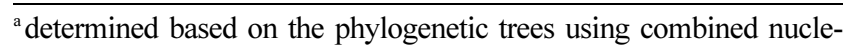
otide sequences of EF1A and RPB2.

UPGMA and NJ trees. Unlike the case of F. fujikuroi, those residing in the distinct subclades were not host-specific. Among the clade of $F$. commune in 2010, two potential subclades were identified; one was for eight isolates, with $74 \%$ and $59 \%$ BS in the UPGMA and NJ trees, respectively, and the other included 12 isolates and one reference strain with $81 \%$ and $41 \%$ BS in the UPGMA and NJ trees, respectively (Fig. 2). Unlike these species, no distinct subclade was identified in $F$. verticillioides from either 2009 or 2010.

Determination of mating types among the fungal isolates. Generally, more than $80 \%$ of the G. fujikuroi species

Table 2. Distribution of mating types ${ }^{\mathrm{a}}$ among the putative fumonisin-producing Fusarium isolates obtained from Korean rice and corn in 2009-2010.

\begin{tabular}{|c|c|c|c|c|c|c|c|c|}
\hline \multicolumn{9}{|c|}{ Number of isolates belonging to each $M A T$ / total number of isolates tested from the same host } \\
\hline \multirow{3}{*}{ Species } & \multicolumn{4}{|c|}{2009} & \multicolumn{4}{|c|}{2010} \\
\hline & \multicolumn{2}{|c|}{ Rice } & \multicolumn{2}{|c|}{ Corn } & \multicolumn{2}{|c|}{ Rice } & \multicolumn{2}{|c|}{ Corn } \\
\hline & MAT1-1 & MAT1-2 & MAT1-1 & MAT1-2 & MAT1-1 & MAT1-2 & MAT1-1 & MAT1-2 \\
\hline F. fujikuroi & $12 / 18$ & $6 / 18$ & $8 / 9$ & $1 / 9$ & $28 / 31$ & $3 / 31$ & $12 / 13$ & $1 / 13$ \\
\hline F. proliferatum & $1 / 2$ & $1 / 2$ & $0 / 2$ & $2 / 2$ & $5 / 5$ & $0 / 5$ & $9 / 10$ & $1 / 10$ \\
\hline F. verticillioides & nd & nd & $4 / 4$ & $0 / 4$ & $1 / 1$ & $0 / 1$ & $31 / 38$ & $7 / 38$ \\
\hline F. commune & nd & nd & nd & nd & $15 / 17$ & $2 / 17$ & $1 / 1$ & $1 / 0$ \\
\hline F. subglutinans & nd & nd & nd & nd & nd & nd & $4 / 5$ & $1 / 5$ \\
\hline F. concentricum & $1 / 1$ & $0 / 1$ & nd & nd & $2 / 5$ & $3 / 5$ & $1 / 1$ & $0 / 1$ \\
\hline
\end{tabular}

${ }^{a}$ determined by PCR with mating-type-specific primer sets. nd: not determined. 
complex isolates examined, except for those of $F$. concentricum, were confirmed to carry the MAT1-1 locus (Table 2). In F. verticillioides and $F$. subglutians, the ratio of mating types was significantly skewed from $1: 1$, and MAT1-1 was found in $83.7 \%$ and $80.0 \%$, respectively, of the total isolates of the species examined. Similarly, high frequencies of MAT1-1 were detected among the isolates of $F$. fujikuroi and $F$. proliferatum. Forty-eight isolates $(91.0 \%)$ of $F$. fujikuroi were identified as MAT1-1 among the total of 53 isolates examined from corn samples in 2009, and from both rice and corn samples in 2010 . Fourteen $(93.3 \%)$ of the $15 \mathrm{~F}$. proliferatum isolates obtained in 2010 were MAT1-1. However, the frequencies of MAT1-1 among the rice isolates of $F$. fujikuroi in 2009, and the $F$. proliferatum isolates from both rice and corn in 2009, were relatively lower than those among the isolates described above $(66.7 \%$ $(12 / 18)$ and $25 \%(1 / 4)$, respectively). In F. commune, the teleomorph of which is unknown, $16(88.9 \%)$ of the 18 isolates examined in 2010 were identified as MAT1-1.

\section{Discussion}

Although members of the G. fujikuroi species complex are known to be frequently found on rice and/or corn worldwide, this is the first report on the population structure of the putative fumonisin-producing G. fujikuroi species complex associated with rice and corn in Korea. The presence of FUM1, by PCR amplification, was used to narrow down the fungal isolates to be studied here from the Fusarium collections initially selected based only on colony characteristics. However, it cannot validate the species identification within the G. fujikuroi species complex because no species-specific amplification of FUM1 has been achieved (Baird et al., 2008; Jurado et al., 2010). Moreover, the usefulness of the species-specific primers sets used in this study was limited because they showed no specificity in some isolates (data not shown), as described previously (Kang et al., 2011). As many previous studies have suggested, this study showed that a phylogenetic analysis using the nucleotide sequences of a protein-encoding gene is the best way to differentiate G. fujikuroi species complex isolates at the species level. In particular, comparisons of multilocus DNA sequences are more efficient for species identification because the sequence of a single gene, such as $E F 1 A$, is sometimes not sufficiently variable between closely related species such as $F$. verticillioides and $F$. proliferatum (data not shown).

Because most corn plants collected in Gangwon-do were closely surrounded by rice, both crops have likely shared the fungal inoculum in these sampling sites. Despite this, the species compositions in the G. fujikuroi species complex were different in the two plants. The most dramatic host preference was shown in $F$. verticillioides, which was the dominant species on corn although the frequency varied by the year of collection, as reported in Korea and other countries (Chung and Kim 1995; Desjardins et al., 2000; Park et al., 2001; Zainudin et al., 2008). Almost no contamination of $F$. verticillioides in rice was also consistent with the cases of other countries, although contamination frequencies were higher than in this study (Amatulli et al., 2010; Desjardins et al., 2000; Wulff et al., 2010; Zainudin et al., 2008). In contrast, F. fujikuroi, F. proliferatum, and $F$. concentricum were commonly recovered from both rice and corn. The occurrence of $F$. concentricum in cereals here is the first report in Korea, although it had been collected from the Asian brown plant hopper (Nilaparvata lugens) in Korea (Nirenberg and O'Donnell, 1998). Unlike the three species of the G. fujikuroi complex described above, $F$. concentricum has not been investigated for its capabilities as a pathogen to cause plant diseases or to produce fumonisin, although its ability to produce moniliformin has been reported (Vesonder et al., 1995). As a non-member of the $G$. fujikuroi species complex, the recently described $F$. commune was recovered mostly from rice, with a frequency of $29.4 \%$; it is known to be a sister taxon of the F. oxysporum complex and has been isolated from soil and diverse plant substrates (pea, carnation, corn, carrot, barely and conifers) (HaminiKadar et al., 2010; Skovgaard et al., 2003). This is also the first report of the association of $F$. commune with rice grains with a high frequency. However, the capabilities for fumonisin production and pathogenicity toward rice in $F$. commune remain unknown, although damping off and root rot caused by this species have been reported in conifers (Kim et al., 2012).

The major species of the G. fujikuroi species complex recovered in this study are heterothallic for sexual reproduction. If sexually fertile fungal isolates of the two opposite mating types (MAT1-1 and MAT1-2) are equally distributed in the field, genetic exchanges would be expected to occur frequently among field isolates. Previous studies reported the detection of both mating types with an equal (11 MAT1-1:14 MAT1-2) or a slightly skewed ratio (22 MAT1-1:50 MAT1-2) in F. fujikuroi from rice and F. verticillioides on corn, respectively, in Korea (Choi et al., 2009; Park et al., 2001), indicating the possibility of sexual reproduction in the field. However, this study revealed that more than $80 \%$ of the G. fujikuroi species complex examined consisted of the single mating type (MAT1-1), which is rather similar to the MAT ratio (170 MAT1-1:4 MAT1-2) among $F$. fujikuroi isolates from California rice and water grass (Carter et al., 2008). This apparent skewed distribution of the single mating type suggests that asexual reproduction may occur exclusively in Korea, leading to the generation of clonal population structures, probably such as those 
belonging to the subclades within each species in the phylogenetic trees (Figs. 1 and 2), although their BS values were not very high. Additionally, the presence of ricespecific subclades of $F$. fujikuroi and $F$. proliferatum in trees may indicate that these isolates have a long evolutionary relationship with rice in Korea; those isolated from corn were possibly introduced into Korea more recently than rice isolates. This may be comparable to the presence of host-specific polymorphisms of $F$. proliferatum isolates from different host plants in partial sequences of FUM1 (Stepien et al., 2011). However, a comprehensive understanding of genetic diversity and population structures of these species from both crops requires further investigation, such as the determination of effective population size and genetic exchanges of multiloci using more field isolates obtained over longer time periods.

The differences in predominant species in rice and corn suggest that problems possibly associated with these species would be different in the two crops. Relatively high frequencies of $F$. verticillioides and $F$. proliferatum on corn, both of which are known to be high producers of fumonisin, indicate that corn is more likely to be associated with mycotoxicosis caused by fumonisin than rice. F. proliferatum is the second most common species in rice, but the risk of mycotoxin problems in rice would be much lower than in corn because fumonisin accumulated on rice caryopsis, probably due to the $F$. proliferatum contamination, can be removed during the polishing process. This was demonstrated in the case of trichothecenes contamination on rice (Lee et al., 2011). In contrast, the development of bakanae diseases, caused mainly by F. fujikuroi, would be of more importance in rice than problems due to mycotoxin contamination. In this respect, the pathogenicity of F. fujikuroi isolates toward rice should be determined in the future. Most F. fujikuroi isolates obtained here produced only small amount of fumonisins on rice substrate (Lee et al., unpublished data), as in other cases (Desjardins et al., 2000; Wulff et al., 2010; Zainudin et al., 2008).

In conclusion, this study provides insight into understanding the population structure and dynamics of the fumonisin-producing G. fujikuroi species complex recovered from rice and corn in Korea. The species composition and genetic diversity revealed here will be helpful in developing strategies for reducing the risk of disease development and ultimately the contamination of mycotoxins in important cereals in Korea.

\section{Acknowledgments}

This study was carried out with the support of "Cooperative Research Program for Agricultural Science \& Technology Development (Project No. PJ007340)", and by a grant from the Next-Generation BioGreen 21 Program (No. PJ008210), Rural Development Administration, Republic of Korea.

\section{References}

Amatulli, M. T., Spadaro, D., Gullino, M. L. and Garibaldi, A. 2010. Molecular identification of Fusarium spp. associated with bakanae disease of rice in Italy and assessment of their pathogenicity. Plant Pathol. 59:839-844.

Baird, R., Abbas, H. K., Windham, G., Williams, P., Baird, S., Ma, P., Kelley, R., Hawkins, L. and Scruggs, M. 2008. Identification of select fumonisin forming Fusarium species using PCR applications of the polyketide synthase gene and its relationship to fumonisin production in vitro. Int. J. Mol. Sci. 9:554570.

Britz, H., Coutinho, T. A., Wingfield, M. J., Marasas, W. F., Gordon, T. R. and Leslie, J. F. 1999. Fusarium subglutinans f. sp. pini represents a distinct mating population in the Gibberella fujikuroi species complex. Appl. Environ. Microbiol. 65:11981201.

Carter, L. L. A., Leslie, J. F. and Webster, R. K. 2008. Population structure of Fusarium fujikuroi from California rice and water grass. Phytopathology 98:992-998.

Cerda-Olmedo, E., Fernandez-Martin, R. and Avalos, J. 1994. Genetics and gibberellin production in Gibberella fujikuroi. Antonie Van Leeuwenhoek 65:217-225.

Choi, H. W., Kim, J. M., Hong, S. K., Kim, W. G., Chun, S. C. and $\mathrm{Yu}, \mathrm{S}$. H. 2009. Mating types and optimum culture conditions for sexual state formation of Fusarium fujikuroi isolates. Mycobiology 37:247-250.

Chulze, S. N., Ramirez, M. L., Torres, A. and Leslie, J. F. 2000. Genetic variation in Fusarium section Liseola from no-till maize in Argentina. Appl. Environ. Microbiol. 66:5312-5315.

Chung, S. H. and Kim, Y. B. 1995. Natural occurrence of fumoni$\sin \mathrm{B}_{1}$ in Korean corn and rough rice. Food Sci. Biotechnol. 4: 212-216.

Desjardins, A. E. 2006. Fusarium mycotoxin: chemistry, genetics and biology. APS Press, St. Paul, USA.

Desjardins, A. E., Manandhar, H. K., Plattner, R. D., Manandhar, G. G., Poling, S. M. and Maragos, C. M. 2000. Fusarium species from Nepalese rice and production of mycotoxins and gibberellic acid by selected species. Appl. Environ. Microbiol. 66: 1020-1025.

Desjardins, A. E., Plattner, R. D. and Nelson, P. E. 1997. Production of fumonisin $\mathrm{B}_{1}$ and moniliformin by Gibberella fujikuroi from rice from various geographic areas. Appl. Environ. Microbiol. 63:1838-1842.

Hamini-Kadar, N., Edel-Hermann, V., Gautheron, N. and Steinberg, C. 2010. First report of Fusarium commune and Fusarium redolens causing crown and root rot on tomato in Algeria. New Dis. Rep. 22:3.

Hong, S. Y., Kang, M. R., Cho, E. J., Kim, H. K. and Yun, S. H. 2010. Specific PCR detection of four quarantine Fusarium species in Korea. Plant Pathology J. 26:409-416.

Hsuan, H. M., Salleh, B. and Zakaria, L. 2011. Molecular Identification of Fusarium species in Gibberella fujikuroi species 
complex from rice, sugarcane and maize from peninsular Malaysia. Int. J. Mol. Sci. 12:6722-6732.

Jurado, M., Marin, P., Callejas, C., Moretti, A., Vazquez, C. and Gonzalez-Jaen, M. T. 2010. Genetic variability and fumonisin production by Fusarium proliferatum. Food Microbiol. 27: $50-57$.

Kang, M. R., Kim, J. H., Lee, S. H., Ryu, J. G., Lee, T. and Yun, S. H. 2011. Detection of Fusarium verticillioides contaminated in corn using a new species-specific primer. Res. Plant Dis. (in Korean) 17:369-375.

Kedera, C. J., Plattner, R. D. and Desjardins, A. E. 1999. Incidence of Fusarium spp. and levels of fumonisin $\mathrm{B}_{1}$ in maize in western Kenya. Appl. Environ. Microbiol. 65:41-44.

Kim, E. K., Kim, Y. B., Shon, D. H., Ryu, D. and Chung, S. H. 1998. Natural occurrence of fumonisin $B_{1}$ in Korean rice and its processed foods by enzyme-linked immunosorbent assay. Food Sci. Biotechnol. 7:221-224.

Kim, M.-S., Stewart, J. E., Dumroese, R. K. and Klopfenstein, N. B. 2012. Occurrence of the root rot pathogen, Fusarium commune, in forest nurseries of the midwestern and western United States. J. Phytopathol. 160:112-114.

Lee, T., Lee, S. H., Shin, J. Y., Yun, J. C., Lee, Y. W. and Ryu, J. G. 2011. Occurrence of Fusarium mycotoxins in rice and its milling by-products in Korea. J. Food Prot. 74:1169-1174.

Leslie, J. F. 1991. Mating populations in Gibberella fujikuroi (Fusarium section Liseola). Phytopathology 81:1058-1060.

Leslie, J. F. 1999. Genetic status of the Gibberella fujikuroi species complex. Plant Pathology J. 15:259-269.

Leslie, J. F., Zeller, K. A., Logrieco, A., Mule, G., Moretti, A. and Ritieni, A. 2004. Species diversity of and toxin production by Gibberella fujikuroi species complex strains isolated from native prairie grasses in Kansas. Appl. Environ. Microbiol. 70: 2254-2262.

Liu, Y. L., Whelen, S. and Hall, B. D. 1999. Phylogenetic relationships among ascomycetes: evidence from an RNA polymerase II subunit. Mol. Biol. Evol. 16:1799-1808.

Marasas, W. F. O., Thiel, P. G., Rabie, C. J., Nelson, P. E. and Toussoun, T. A. 1986. Moniliformin production in Fusarium section Liseola. Mycologia 78:242-247.

Mule, G., Susca, A., Stea, G. and Moretti, A. 2004. A species-specific PCR assay based on the calmodulin partial gene for identification of $F$. verticillioides, F. proliferatum and $F$. subglutinas. Eur. J. Plant Pathol. 110:495-502.

Nirenberg, H. I. and O'Donnell, K. 1998. New Fusarium species and combinations within the Gibberella fujikuroi species complex. Mycologia 90:434-458.

O'Donnell, K., Cigelnik, E. and Nirenberg, H. I. 1998. Molecular systematics and phylogeography of the Gibberella fujikuroi species complex. Mycologia 90:465-493.

O'Donnell, K., Nirenberg, H. I., Aoki, T. and Cigelnik, E. 2000. A Multigene phylogeny of the Gibberella fujikuroi species complex: Detection of additional phylogenetically distinct species. Mycoscience 41:61-78.

Park, S. Y., Seo, J. A., Lee, Y. W. and Lee, Y. H. 2001. Population genetic analyses of Gibberella fujikuroi isolates from maize in
Korea. Plant Pathology J. 17:281-289.

Proctor, R. H., Desjardins, A. E., Plattner, R. D. and Hohn, T. M. 1999. A polyketide synthase gene required for biosynthesis of fumonisin mycotoxins in Gibberella fujikuroi mating population A. Fungal Genet. Biol. 27:100-112.

Proctor, R. H., Plattner, R. D., Brown, D. W., Seo, J. A. and Lee, Y. W. 2004. Discontinuous distribution of fumonisin biosynthetic genes in the Gibberella fujikuroi species complex. Mycol. Res. 108:815-822.

Rahjoo, V., Zad, J., Javan-Nikkhah, M., Mirzadi Gohari, A., Okhovvat, S. M., Bihamat, M. R., Razzaghian, J. and Klemsdal, S. S. 2008. Morphological and molecular identification of Fusarium isolated from maize ears in Iran. J. Plant Pathol. 90: 463-468.

Rheeder, J. P., Marasas, W. F. and Vismer, H. F. 2002. Production of fumonisin analogs by Fusarium species. Appl. Environ. Microbiol. 68:2101-2105.

Seo, J. A. and Lee, Y. W. 1999. Natural occurrence of the C series of fumonisins in moldy corn. Appl. Environ. Microbiol. 65: 1331-1334.

Skovgaard, K., Rosendahl, S., O'Donnell, K. and Nirenberg, H. I. 2003. Fusarium commune is a new species identified by morphological and molecular phylogenetic data. Mycologia 95: 630-636.

Son, S. W., Nam, Y. J., Lee, S. H., Lee, S. M., Lee, S., Kim, M., Lee, T., Yun, J. C. and Ryu, J. G. 2011. Toxigenic fungal contaminants in the 2009-harvested rice and its milling-byproducts samples collected from rice processing complexes in Korea. Res. Plant Dis. (in Korean) 17:280-287.

Steenkamp, E. T., Wingfield, B. D., Coutinho, T. A., Zeller, K. A., Wingfield, M. J., Marasas, W. F. and Leslie, J. F. 2000. PCRbased identification of MAT-1 and MAT-2 in the Gibberella fujikuroi species complex. Appl. Environ. Microbiol. 66:43784382.

Stepien, L., Koczyk, G. and Waskiewicz, A. 2011. Genetic and phenotypic variation of Fusarium proliferatum isolates from different host species. J. Appl. Genet. 52:487-496.

Thompson, J. D., Higgins, D. G. and Gibson, T. J. 1994. CLUSTALW: improving the sensitivity of progressive multiple sequence alignment through sequence weighting, positionspecific gap penalties and weight matrix choice. Nucleic Acids Res. 22:4673-4680.

Vesonder, R. F., Logrieco, A., Bottalico, A., Altomare, C. and Peterson, S. W. 1995. Fusarium species associated with banana fruit rot and their potential toxigenicity. Mycol. Res. 101:437445.

Wulff, E. G., Sørensen, J. L., Lübeck, M., Nielsen, K. F., Thrane, U. and Torp, J. 2010. Fusarium spp. associated with rice bakanae: ecology, genetic diversity, pathogenicity and toxigenicity. Environ. Microbiol. 12:649-657.

Zainudin, L. A. I. M., Razak, A. A. and Salleh, B. 2008. Secondary metabolite profiles and mating populations of Fusarium species in section Liseola associated with bakanae disease of rice. Malaysian J. Microbiol. 4:6-13. 